\title{
Microvascular Architecture of the Mouse Urinary Bladder Described with Vascular Corrosion Casting, Light Microscopy, SEM, and TEM
}

\author{
F. Hossler*, A. Lametschwandtner**, R. Kao***, C. Bills*, and F. Finsterbusch** \\ *East Tennessee State University College of Medicine, Department of Anatomy \& Cell Biology, \\ Johnson City, TN 37614 USA \\ **University of Salzburg, Department of Organismic Biology, Vascular and Muscle Research Unit, \\ Hellbrunnerstrasse 34, A-4020 Salzburg, Austria \\ ***East Tennessee State University College of Medicine, Department of Surgery, Johnson City, \\ TN 37614 USA
}

The urinary bladder is a unique organ in that its normal function includes filling and emptying, and changes in pressure on the vasculature of the wall. It has been of interest to learn if any unique anatomical features of its vasculature exist that help maintain blood flow despite these changes in internal pressure, which may compromise blood flow in these vessels. Although the microvasculature of the urinary bladder in the rabbit [1] and dog [2] have been previously described, information on the mouse bladder vasculature could be especially valuable considering the variety of genetic models that have recently been developed for this species.

For routine microscopy, bladders were fixed in $0.1 \mathrm{M}$ cacodylate buffered, formaldehydeglutaraldehyde (1\%-2.5\%) mixtures ( $\mathrm{pH} 7.4)$, then embedded and sectioned from Epon-Araldyte resin blocks. For vascular corrosion casts, the bladder vasculature were first flushed at manual pressure with warm physiological saline via the abdominal or thoracic aorta, then perfused with Mercox CL2-B resin (Ladd Res. Inc, Burlington, VT) or Mercox Cl-2B resin diluted with 20\% methyl methacrylate (Sigma Chemical Co., St. Louis. MO).

The layers of the bladder wall (Figs. 1-3) include a thin serosa, a substantial muscularis consisting of smooth muscle arranged in opposing layers, a very loosely organized and expandable submucosa consisting of collagen fibers and ground substance, and a mucosa consisting of a dense, collagenous lamina propria lined with a transitional epithelium. Folding of the bladder mucosa and the thickness of each layer of the wall (especially the submucosa), change dramatically with bladder distension and relaxation.

Although it is well known that the vasculature of the mouse varies considerably within the species [3], most commonly the urinary bladder is supplied by left and right vesicular branches of the urogenital arteries [3]. Each vesicular artery has dorsal and ventral divisions which enter the bladder wall basally, adjacent to the ureteral and urethral vasculatures (Figs. 4 and 5). The vesicular arteries spread from the base (dorsal end) of the bladder toward its apex (ventral end), and are highly coiled, especially in the relaxed bladder (Figs. 4 and 6). Branches (also coiled) of the vesicular arteries penetrate the bladder wall to supply a very dense mucosal capillary bed lying just deep to the lining epithelium (Figs. 5-9). Arterial sphincters are evident at the branch points of some of the arterioles supplying the capillary bed (Fig. 7). The mucosal capillary bed of the bladder is characterized by extensive branching and anastomoses (Figs. 8 and 9), and is continuous with a similar arrangement in the ureters and the urethra. The wall of the urethra contains a very 
dense mass of longitudinal veins and arteries (Fig. 5) that appear to be continuous with the supply vessels in the bladder wall, but the contribution of this vasculature to the blood supply of the bladder or vise versa has not as yet been determined.

During filling of the urinary bladder, it appears likely that blood flow in the wall is facilitated by the extensive coiling of supply vessels, by the abundant branching and anastomoses seen in the mucosal capillary bed as well as in the supply vessels, and perhaps also by the arterial sphincters.

References

[1] F.E. Hossler and F.C. Monson, Anat Rec 243 (1995) 438-448

[2] F.E. Hossler and R.L. Kao, Microsc Microanal 13 (2007) 220-227

[3] M.J. Lopez-Fuster, et al., Anat Histol Embryo 22 (1993) 279-287.

[4] The authors wish to acknowledge the assistance of S. Tholo** with the SEM work.
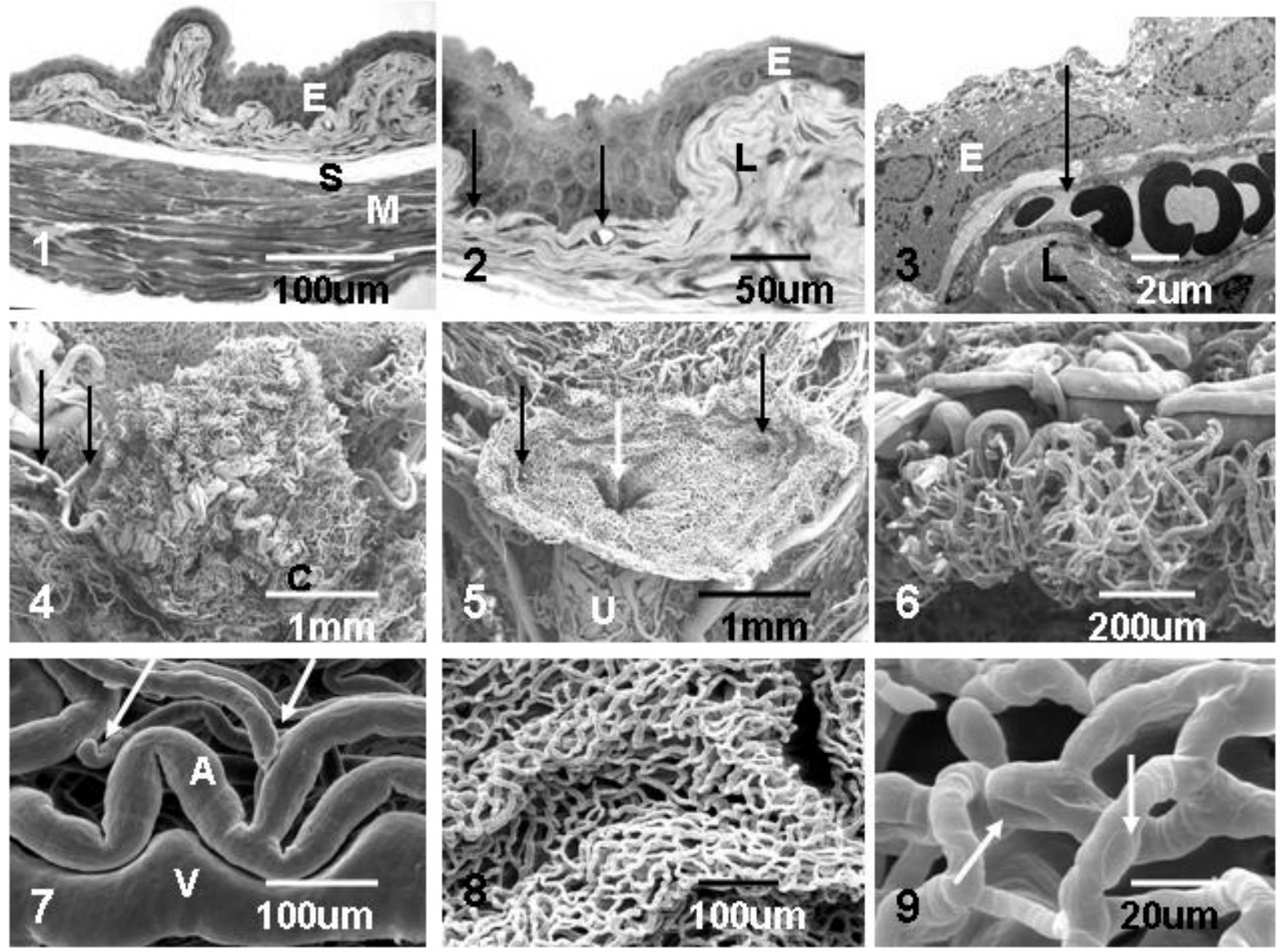

Figs.1-3. Cross sections of lateral bladder wall showing layers (E, epithelium; L, lamina propria; S, submucosa; M, muscularis) and capillaries (arrows). Fig.4. Corrosion cast of bladder, apical view (arrows, vesicular arteries; C, coiled supply vessels). Fig.5. Corrosion cast of bladder cut open to show urethral opening (white arrow), ureteral openings (black arrows), and urethral vasculature (U). Fig.6. Corrosion cast showing cross section of wall (supply vessels above; mucosal capillary bed below). Fig.7. Cast of submucosal arteries (A) and veins (V). Arrows indicate arterial sphincters. Figs.8 and 9. Details of mucosal capillary bed. Arrows indicate endothelial imprints. 